\title{
Avoiding routine postoperative voiding cystourethrogram: Predicting radiologic success for endoscopically treated vesicoureteral reflux
}

Peter (Zhan Tao) Wang ${ }^{1}$; Ahmed Abdelhalim ${ }^{2,3}$; Arman Walia ${ }^{4}$; Elias Wehbi ${ }^{2}$; Sumit Dave ${ }^{1}$; Antoine Khoury ${ }^{3}$

${ }^{1}$ Department of Surgery, Division of Urology, London Health Sciences Centre, Western University, London, ON, Canada; ${ }^{2}$ Department of Urology, Children's Hospital of Orange County, University of California, Irvine, CA, United States; ${ }^{3}$ Department of Urology, Mansoura Urology and Nephrology Center, Mansoura University, Egypt; ${ }^{4}$ Children's Hospital of Orange County, University of California, Irvine, CA, United States

Cite as: Can Urol Assoc J 2018 November 5; Epub ahead of print. http://dx.doi.org/10.5489/cuaj.5589

Published online November 5, 2018

$* * *$

\section{Abstract}

Introduction: Variability in the success rates for the endoscopic correction of vesicoureteral reflux (VUR) has prompted a debate regarding the use of routine postoperative voiding cystourethrogram (VCUG). This study examines the predictive performance of intraoperative mound morphology (IMM) and the presence of a postoperative ultrasound mound (PUM) on radiologic success, as well as investigates the role of using these two predictive factors as a composite tool to predict VUR resolution after endoscopic treatment.

Methods: This retrospective study included children with primary VUR who underwent endoscopic correction with a double HIT technique and dextranomer/hyaluronic acid copolymer. IMM was assessed intraoperatively. The presence of a PUM and VUR resolution were assessed by postoperative ultrasound (US) and VCUG, respectively. Radiologic success was defined as VUR resolution.

Results: A total of 70 children (97 ureters) were included in the study. The overall radiologic success rate was $83.5 \%$. There was no statistically significant association between radiologic success and IMM (85.2\% with excellent and 87.5\% with “other” morphology; $\mathrm{p}=0.81)$. The sensitivity and specificity of PUM for radiologic success in this study was $98 \%$ and $71 \%$, respectively, while the sensitivity and specificity of the combined prediction model were $81.9 \%$ and $85.7 \%$, respectively.

Conclusions: We objectively demonstrated that IMM was a poor predictor of radiologic success and should be used with caution. In addition, the performance of a combined prediction model 
was inferior to the presence of a PUM alone. As such, selective use of postoperative VCUG may be guided solely by the presence of a PUM.

\section{Introduction}

The endoscopic correction of vesicoureteral reflux (VUR) is a safe and minimally invasive alternative to ureteral reimplantation in a select population. However, unlike the consistently high success rates of $90-100 \%{ }^{1,2}$ with ureteral reimplantation, the success rates of endoscopic repair are highly variable ranging between $44 \%-100 \% .^{3-5}$ Therefore, the American Urological Association (AUA) continues to recommend routine voiding cystourethrogram (VCUG) following endoscopic correction of primary VUR. ${ }^{3}$

However, there is morbidity associate with a VCUG; it is an invasive test that exposes children to ionizing radiation and causes emotional and physical distress. ${ }^{6}$ As such, some authors have advocated for a selective or "watch and see" approach, only performing a postoperative VCUG after a urinary tract infection. ${ }^{7,8}$ In order to circumvent the routine use of postoperative VCUG, a number of clinical and radiological factors have been proposed in the literature to predict the success of endoscopic VUR correction.

The two most extensively studied predictive factors include the intraoperative mound morphology (IMM) and the presence of a postoperative ultrasound mound (PUM). ${ }^{5,9-12}$ However, the evidence for the predictive performance of these two factors are conflicting, as each factor have multiple proposed limitations. ${ }^{13-15}$ We hypothesized that a stratification model combining these two factors may circumvent the limitations of using IMM and PUM individually.

The objectives of this study are to examine the predictive values of IMM and PUM on radiological success; and to test if the combined use of IMM and PUM is superior to either factor in predicting VUR resolution post endoscopic VUR correction.

\section{Methods}

This retrospective study was approved by our institutional review board (IRB \#150856). Children (age less than 18 years) with primary VUR who underwent primary endoscopic correction between October 2008 and October 2013 were screened for inclusion. Patients with neurogenic bladders, posterior urethral valves, ureteroceles, para-ureteral diverticula, previous anti-reflux surgery and those without intraoperative documentation of mound morphology, postoperative US or postoperative VCUG were excluded from the analysis.

\section{Preoperative evaluation}

Preoperative evaluation included a comprehensive history and physical exam with particular attention paid to the presence of bladder and bowel dysfunction (BBD). In toilet-trained children, BBD was diagnosed clinically by the presence of urinary urgency, frequent or infrequent voiding, incontinence and constipation. In non-toilet trained children, BBD was defined as less than 1 bowel movement per day. Patients with BBD were treated aggressively prior to surgical intervention. 
All children included in this study underwent preoperative renal bladder US and VCUG. Preoperative factors such as VUR grade, hydronephrosis, hydroureter and ureteral duplication were documented. The indications for endoscopic correction included breakthrough UTI and persistent VUR despite conservative management.

Surgical technique

After induction of general anesthesia, a cystoscopic survey was performed. The bladder was then filled to half capacity and the ureters were hydrodistended. All children were treated endoscopically using the double hydrodistension-implantation technique (Double HIT) with dextranomer/hyaluronic acid copolymer (Deflux ${ }^{\circledR}$, Q-Med Scandinavia) as previously described. ${ }^{16}$

The injected volume in each ureter and IMM were recorded. Excellent IMM was strictly defined as having a characteristic "volcano" appearance with a coapted ureteral orifice at the summit that did not hydrodistend (Figure 1A), ${ }^{16,17}$ while 'other' morphology was any appearance that did not fit this definition. The injected volume per ureter was stratified into $<1 \mathrm{~mL}$ and $>1$ $\mathrm{mL}$ groups. Children were discharged home on antibiotic prophylaxis until a postoperative VCUG was obtained at 12 weeks.

\section{Followup}

Patients underwent postoperative renal bladder US and VCUG at 6 and 12 weeks, respectively. Postoperative imaging was reviewed first by pediatric radiologists, and again by the authors (ZW and AW). The presence of a postoperative mound on bladder US was defined as an echogenic nodule at the site of the ureterovesical junction (Figure 1B and 1C). Radiologic success was defined by the absence of VUR on postoperative VCUG. Postoperative urinary tract infections (febrile/symptomatic) were documented on follow-up.

\section{Statistics}

The combined prediction model was defined as follows: children with both an excellent IMM and the presence of a PUM were categorized into the favorable group; whereas those with 'other' IMM and/or the absence of a PUM were considered unfavorable. Demographic, intraoperative and postoperative variables were compared using the Mann-Whitney U test for continuous variables and Fischer's exact test for categorical variables. Sensitivity, specificity, positive predictive value (PPV) and negative predictive value (NPV) were calculated and presented as a percentage. Statistical analysis was performed with SPSS Statistics for Windows (Version 24.0. Armonk, NY: IBM Corp.). Statistical significance was set at $\mathrm{p}<0.05$ with all reported $\mathrm{p}$ values being two-sided.

\section{Results}

A total of 101 consecutive children treated endoscopically for VUR met the inclusion criteria. Thirty-one children were excluded due to prior surgery or incomplete data. The remaining 70 
children with 97 refluxing ureters were included in the final analysis. Demographic data of the study population is summarized in Table 1 . The mean age at surgery was $4.2 \pm 3.4$ years with 58 females and 12 males. The mean follow-up was $22.6 \pm 2.3$ months with a median of 16.1 (range 2.6 - 75.7) months. There were 71 (73.1\%) ureters with low grade VUR (I-III) and 26 (26.8\%) with high grade VUR (IV-V). VUR was bilateral in 27 children (55.7\% of ureters) and BBD was present preoperatively in 27 (32.5\%).

Preoperative, intraoperative, and postoperative factors were compared and summarized in Table 2. The overall radiologic success rate for the study population was 83.5\%. Excellent IMM was observed in 81 (83.5\%) ureters, while PUM was visualized in 85 (87.6\%) ureters on the first follow-up US obtained at a median of 42 days (range 12 - 112 days) postoperatively. Higher radiologic success $(95.3 \%$ versus $8.3 \%, \mathrm{p}<0.001)$ was demonstrated in the presence of a PUM. Additionally, this study found that none of the children with a PUM who had persistent VUR postoperatively developed a recurrent urinary tract infection within the follow-up period.

The radiologic success rate was significantly higher in children with low grade VUR compared to high grade $(91.5 \%$ and $69.2 \%, p=0.031)$. Meanwhile, radiologic success was statistically less common in males compared to females $(62.5 \%$ versus $88.9 \%, p=0.023)$. There was no statistically significant association between radiologic success and IMM (85.2\% with excellent and $87.5 \%$ with 'other' morphology showed VUR resolution, $\mathrm{p}=0.81$ ) or injected volume (80.6\% and 91.3\%, p=0.16). Similarly, there was no differences between the radiologic success of children with or without BBD (91.1\% vs. 81.5\%, p=0.282), although there was a trend towards improved success in the absence of BBD.

Table 3 compares the sensitivity and specificity of PUM for radiological success between our study and those in the literature. In this study, the presence of a PUM was 98\% sensitive and $71 \%$ specific for radiologic success. In our proposed combined prediction model, the favorable group had a significantly higher radiologic success rate than the unfavorable group (97.1\% versus 55.6\%, $\mathrm{p}<0.001$ ); the sensitivity, specificity, PPV and NPV of the combined prediction model were $81.9 \%$ and $85.7 \%, 97.1 \%$ and $44.4 \%$ respectively. The predictive performance of IMM, PUM and our combined prediction model is summarized in Table 4. While the combination of IMM and PUM has improved specificity and PPV over PUM, PUM had superior sensitivity and NPV when used in isolation.

\section{Discussion}

O’Donnell et al. first described the endoscopic correction of VUR in $1984{ }^{18}$ The procedure has since become an effective alternative to ureteral reimplantation. ${ }^{19,20}$ The AUA VUR Guideline Update Committee, extracting outcome data from 131 articles involving 17,972 patients reported an overall radiologic success rate of $83 \% .^{3}$ This correlates well with our overall radiological success rate of $83.5 \%$. However, more importantly the AUA committee found that the success rates for endoscopic VUR correction varies widely depending on the definition of treatment 
success, as wells as with various preoperative and intraoperative factors. ${ }^{21-23}$ This prompted the AUA to recommend routine postoperative VCUG after endoscopic correction.

Given that a VCUG is not a benign procedure, some authors have advocated for selective use of postoperative VCUG based on factors that predict treatment success ${ }^{21}$ or forego a postoperative VCUG until the patient develops a repeat urinary tract infection. ${ }^{7,8}$ Multiple studies have investigated the predictive value of IMM and PUM visualization to circumvent the need for routine postoperative VCUG; however the results have been conflicting. ${ }^{5,7-12}$

Excellent IMM has been cited as an index for technical success since the inception of endoscopic VUR correction. ${ }^{18}$ Following FDA approval of dextranomer/hyaluronic acid copolymer, Lavelle et al. retrospectively examined 52 children who underwent endoscopic correction of VUR with the STING technique and this novel bulking agent. The authors found higher success rates when IMM had a "volcano" appearance compared to "other” morphology (87\% versus 53\%, $\mathrm{p}=0.004) .{ }^{10}$ Similarly, Yucel et al. concluded that a satisfactory mound was the most important factor predicting radiologic success in children undergoing endoscopic correction with either a STING or HIT technique. The authors demonstrated an $81 \%$ success rate for satisfactory intraoperative mounds compared to 36\% for “other” morphologies ( $<<0.0001)$; and, using multivariate logistic regression, showed that a satisfactory mound was 11.5 times more likely to succeed (OR 11.5; 95 CI 5.6 - 23.8, p <0.0001). ${ }^{9}$

However, the adequacy of IMM is often plagued by subjectivity and possibly assessor bias. This was demonstrated in a survey of 96 pediatric urologists using 15 intraoperative mound images by Hidas et al., who reported an average probability of $66 \%$ in predicting radiologic success or failure based on morphology alone. ${ }^{15}$ Some proposed explanations for the discrepancies between IMM and radiologic outcomes include postoperative mound shrinkage, reshaping, and migration. ${ }^{11,13,15}$

Several authors have also investigated the value of PUM visualization as a predictor for radiologic success. It is postulated that the presence of a mound on postoperative imaging implies positional stability and that mound characteristics would be indicative of retained volume. In one of the earliest reports, Herz et al. reported that the detection of Polydimethysiloxane (Macroplastique) implants on follow-up US was $89 \%$ sensitive and $86 \%$ specific for successful correction of VUR. ${ }^{24}$ Lee et al., on multivariate analysis, demonstrated an odds ratio of 6.05 (CI 2.99 - 12.21, p < 0.001) for radiological success with the presence of a PUM. $^{12}$ The authors reported a sensitivity and specificity of $79.5 \%$ and $59.3 \%$, respectively. McMann et al. examining 986 ureters showed that the presence of a PUM had a sensitivity of $90 \%$ and specificity of $27 \%$ for radiologic success.

Conversely, some have refuted the predictive value of a PUM. VUR resolution was not statistically different among patients with or without PUM in a study by Ellsworth et al. ${ }^{25}$ However, it is difficult to generalize these findings as the authors did not report the technique used and included children with repeat procedures. Likewise, Zamilpa et al. reported poor sensitivity (38\%), specificity (67\%), and overall accuracy (55\%) of PUM as a predictor of 
successful endoscopic correction. ${ }^{14}$ Lastly, a long-term study by Ozcan et al. evaluated the performance of a PUM in predicting radiologic success after endoscopic correction with a STING technique. The authors concluded that PUM was not a reliable predictor due to mound shrinkage with time (mean follow-up of 2.2 years). ${ }^{26}$

Additionally, the predictive performance of various characteristics of a PUM on radiologic success have been investigated. Zambaiti et al. reported that PUM height was predictive of radiologic success with a sensitivity and specificity of $100 \%$ and $66 \%$, respectively, using a height cut off of $9.8 \mathrm{~mm} .{ }^{27}$ Park et al. graded the PUM (1-3) and found more pronounced and protruding PUMs to be more predictive of radiologic success. ${ }^{28}$ More recently, the use of an intraoperative US-assisted approach for endoscopic VUR correction was investigated. The authors found that this approach led to increased intraoperative accuracy in positioning the bulking agent, as well as improved a surgeon's ability to achieve an ideal IMM. ${ }^{29}$ However, all these variations on the use of an US mound to predict endoscopic success requires dedicated US protocols, global acceptance by radiologists and/or specialized equipment.

The stratification of children to avoid routine postoperative VCUG have been previously investigated. ${ }^{7,21}$ Arlen et al. studied 222 children and stratified them into low or high-risk groups for treatment failure using preoperative clinical and radiologic risk factors. Children aged less than 2 years, with $\geq 3$ preoperative febrile UTIs, documented BBD and those with grades IV-V VUR were classified as high risk for treatment failure. ${ }^{21}$ The authors found that children with any of the aforementioned risk factors were 13 times more likely to have radiological failure. However, unlike Arlen et al., this study investigated the combination of a procedural (IMM) and postoperative (PUM) factor rather than preoperative risk factors.

Overall, the majority of these previous studies evaluating the predictive performance of IMM and PUM on radiologic success includes a significant proportion of children treated with a STING technique; as well as the use of varying bulking agents. In this study, we examine a homogenous population of children with primary VUR treated exclusively with a double HIT technique using dextranomer/hyaluronic acid copolymer. Although the sensitivity and specificity vary widely between studies examining the predictive role of PUM (sensitivity ranged between 38 to $100 \%$, while specificity ranged from 22 to $67 \%$ ), our results (sensitivity $98 \%$ and specificity 71\%) fell within these previously reported ranges.

Furthermore, to our knowledge, this study is the first to objectively demonstrate a poor correlation between an excellent IMM and radiologic success (87.5\% of mounds observed as "other" were eventually found to be successfully treated). In this study, we also explored the use of a combined prediction model comprising both the presence of a PUM and excellent IMM to predict radiologic success. Although we demonstrated a higher radiologic success rate in children classified into the favorable versus unfavorable group $(97.1 \%$ versus $55.6 \%, \mathrm{p}<0.001)$, the sensitivity of this combined prediction model was lower than the use of PUM alone (82\% versus 98\%, respectively); implying that the model was an inferior screening tool, which may be largely due to the poor predictive performance of IMM. 
What we found in our study was excellent radiologic success when a PUM was present versus absent (95.3\% versus $8.3 \%, \mathrm{p}<0.001)$; a success rate of $95.3 \%$ based on ureters with a PUM is comparable to the success rates of ureteral reimplantation. Following the same rationale for excluding a postoperative VCUG in ureteral reimplantation, one may argue that we can selectively forgo routine postoperative VCUG in children with PUM post endoscopic correction. Furthermore, the presence of a PUM showed excellent sensitivity and PPV in predicting radiologic success. Based on the results of this study, if the use of postoperative VCUG was limited to individual ureters without a PUM, the morbidity of a VCUG would be spared 87.6\% of the time while only missing $4.7 \%$ of ureters that had radiologic failure. If these children subsequently develop a urinary tract infection, a VCUG may be performed at that time.

\section{Limitations}

The interpretation of this study requires consideration of several limitations. The retrospective nature introduces inherent bias (e.g. detection bias); this is compounded by the relatively small sample size, which also predisposes the results to type I and type II errors. The small sample size also limits our ability to perform subgroup analyses and logistic regression.

Furthermore, although the subjectivity of an ideal IMM may never be eliminated, this was minimized in this study with the use of a strict definition for excellent appearance; the high proportion of excellent IMM was the result of an active effort by the surgeon to achieve this appearance through repeated injections. Lastly, as there are no dedicated US protocols in our institution, postoperative ultrasounds were performed without specific indications to locate PUM, as such less obvious PUM may have been missed.

\section{Conclusion}

This study examined the predictive performance of IMM and PUM, as well as a combined prediction model on children with primary VUR corrected using a double HIT technique with dextranomer/hyaluronic acid copolymer. We found IMM to be a poor predictor of radiological success and should be used with caution as an index for technical success. As a result of the poor predictive value of IMM, a combined prediction model was not found to be a useful screening tool to predict radiologic success compared to the presence of PUM alone. Although prospective studies will be required for more definitive conclusions, our study suggests that selective utilization of postoperative VCUG may be guided solely by the presence of a PUM. 


\section{References}

1. Fung LC, McLorie GA, Jain U, et al. Voiding efficiency after ureteral reimplantation: A comparison of extravesical and intravesical techniques. J Urol 1995;153:1972-5.

2. Houle AM, McLorie GA, Heritz DM, et al. Extravesical nondismembered ureteroplasty with detrusorrhaphy: A renewed technique to correct vesicoureteral reflux in children. $J$ Urol 1992;148:704-7.

3. Peters CA, Skoog SJ, Arant BS, Jr., et al. Summary of the aua guideline on management of primary vesicoureteral reflux in children. J Urol 2010;184:1134-44.

4. Kirsch AJ, Perez-Brayfield MR, Scherz HC. Minimally invasive treatment of vesicoureteral reflux with endoscopic injection of dextranomer/hyaluronic acid copolymer: The children's hospitals of atlanta experience. J Urol 2003;170:211-5.

5. Routh JC, Reinberg Y. Predicting success in the endoscopic management of pediatric vesicoureteral reflux. Urology 2010;76:195-8.

6. Nelson CP, Chow JS, Rosoklija I, et al. Patient and family impact of pediatric genitourinary diagnostic imaging tests. J Urol 2012;188:1601-7.

7. Haid B, Berger C, Roesch J, et al. Persistence and recurrence of vesicoureteric reflux in children after endoscopic therapy - implications of a risk-adapted follow-up. Cent European J Urol 2015;68:389-95.

8. Kaye JD, Srinivasan AK, Delaney C, et al. Clinical and radiographic results of endoscopic injection for vesicoureteral reflux: Defining measures of success. J Pediatr Urol 2012;8:297-303.

9. Yucel S, Gupta A, Snodgrass W. Multivariate analysis of factors predicting success with dextranomer/hyaluronic acid injection for vesicoureteral reflux. J Urol 2007;177:1505-9.

10. Lavelle MT, Conlin MJ, Skoog SJ. Subureteral injection of deflux for correction of reflux: Analysis of factors predicting success. Urology 2005;65:564-7.

11. McMann LP, Scherz HC, Kirsch AJ. Long-term preservation of dextranomer/hyaluronic acid copolymer implants after endoscopic treatment of vesicoureteral reflux in children: A sonographic volumetric analysis. J Urol 2007;177:316-20; discussion 20.

12. Lee DG, Lee SW, Park KH, et al. Significance of an endoscopically injected nodule detected on ultrasound as a predictive factor for the resolution of vesicoureteral reflux. Exp Ther Med 2015;9:1058-62.

13. Higham-Kessler J, Reinert SE, Snodgrass WT, et al. A review of failures of endoscopic treatment of vesicoureteral reflux with dextranomer microspheres. J Urol 2007;177:7104; discussion 4-5.

14. Zamilpa I, Koyle MA, Grady RW, et al. Can we rely on the presence of dextranomerhyaluronic acid copolymer mounds on ultrasound to predict vesicoureteral reflux resolution after injection therapy? J Urol 2011;185:2536-41. 
15. Hidas G, Soltani T, Watts B, et al. Is the appearance of the dextranomer/hyaluronic acid mound predictive of reflux resolution? J Urol 2013;189:1882-5.

16. Cerwinka WH, Scherz HC, Kirsch AJ. Dynamic hydrodistention classification of the ureter and the double hit method to correct vesicoureteral reflux. Arch Esp Urol 2008;61:882-7.

17. Puri P, Chertin B, Velayudham M, et al. Treatment of vesicoureteral reflux by endoscopic injection of dextranomer/hyaluronic acid copolymer: Preliminary results. $J$ Urol 2003;170:1541-4; discussion 4.

18. O'Donnell B, Puri P. Treatment of vesicoureteric reflux by endoscopic injection of teflon. Br Med J (Clin Res Ed) 1984;289:7-9.

19. Kirsch AJ, Arlen AM. Evaluation of new deflux administration techniques: Intraureteric hit and double hit for the endoscopic correction of vesicoureteral reflux. Expert Rev Med Devices 2014;11:439-46.

20. Puri P, Kutasy B, Colhoun E, et al. Single center experience with endoscopic subureteral dextranomer/hyaluronic acid injection as first line treatment in 1,551 children with intermediate and high grade vesicoureteral reflux. J Urol 2012;188:1485-9.

21. Arlen AM, Scherz HC, Filimon E, et al. Is routine voiding cystourethrogram necessary following double hit for primary vesicoureteral reflux? J Pediatr Urol 2015;11:40 e1-5.

22. Lorenzo AJ, Pippi Salle JL, Barroso U, et al. What are the most powerful determinants of endoscopic vesicoureteral reflux correction? Multivariate analysis of a single institution experience during 6 years. J Urol 2006;176:1851-5.

23. Capozza N, Caione P. Dextranomer/hyaluronic acid copolymer implantation for vesicoureteral reflux: A randomized comparison with antibiotic prophylaxis. J Pediatr 2002;140:230-4.

24. Herz D, Hafez A, Bagli D, et al. Efficacy of endoscopic subureteral polydimethylsiloxane injection for treatment of vesicoureteral reflux in children: A north american clinical report. J Urol 2001;166:1880-6.

25. Ellsworth PI, Yates JK, Caldamone AA. Presence of dextranomer-hyaluronic acid (dxha) mound on postoperative ultrasound does not predict resolution of vesicoureteral reflux. $J$ Pediatr Urol 2011;7:438-40.

26. Ozcan C, Ergun R, Ozbek SS, et al. Bladder ultrasound in the evaluation of the efficacy of dextranomer/hyaluronic acid injection for treatment of vesicoureteral reflux. J Clin Ultrasound 2007;35:357-62.

27. Zambaiti E, Pensabene M, Montano V, et al. Ultrasonographic mound height as predictor of vesicoureteral reflux resolution after endoscopic treatment in children. J Pediatr Surg 2016;51:1366-9.

28. Park KJ, Jeon TY, Yoo SY, et al. The appearance of dextranomer-hyaluronic acid copolymer implants on ultrasound may predict resolution of vesicoureteral reflux after injection therapy. Clin Radiol 2014;69:939-44. 
29. Zambaiti E, Sergio M, Casuccio A, et al. Intraoperative ultrasound-assisted approach for endoscopic treatment of vesicoureteral reflux in children. J Pediatr Surg 2017;52:1661-5.

30. Kajbafzadeh AM, Aryan Z, Tourchi A, et al. Long-term ultrasound appearance of concomitant autologous blood and dextranomer/hyaluronic acid copolymer implants: Is it associated with successful correction of vesicoureteral reflux? Urology 2013;81:407-13. 


\section{Figures and Tables}

Fig. 1. Intraoperative and postoperative mound assessment. (A) Excellent intraoperative mound morphology with a characteristic volcano-appearance. (B) Ultrasound mound with protrusion into the bladder. $(\boldsymbol{C})$ Ultrasound mound without protrusion into the bladder.
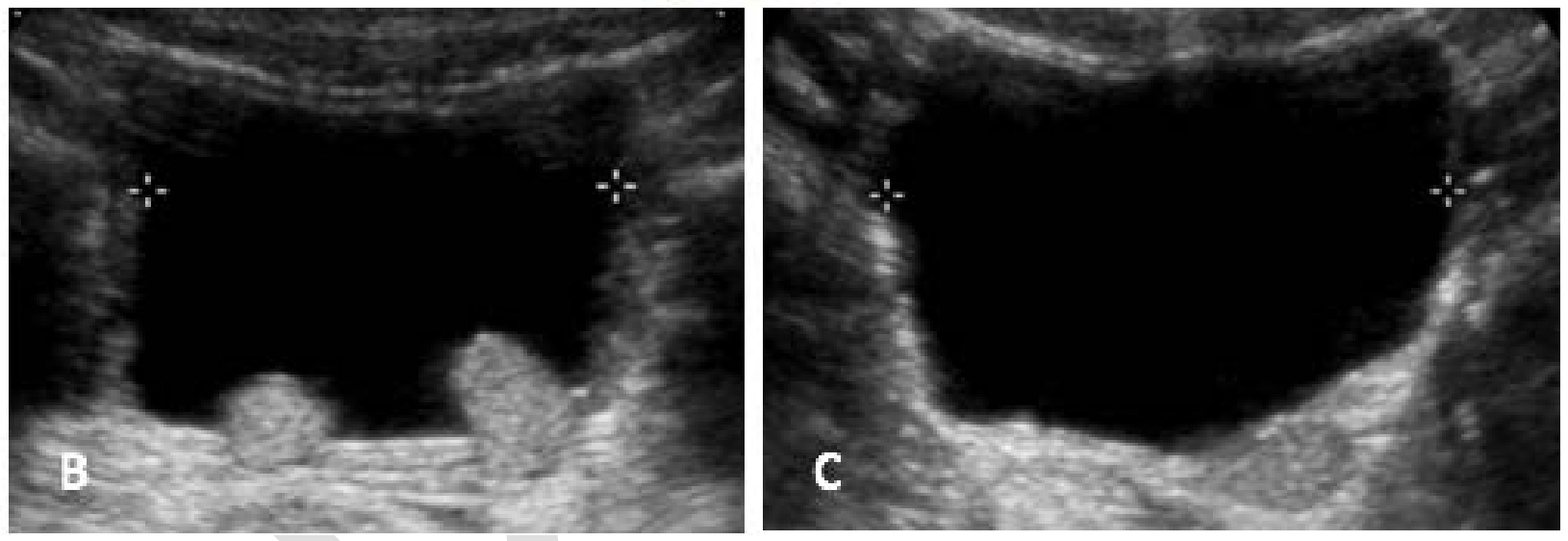


\begin{tabular}{|c|c|c|c|c|}
\hline \multirow[t]{2}{*}{ Variables } & \multirow{2}{*}{$\begin{array}{c}\text { Ureters (\%) } \\
n=97\end{array}$} & \multicolumn{3}{|c|}{ Radiologic success (\%) } \\
\hline & & Success $(n=83)$ & Failure $(n=14)$ & $\mathbf{p}$ \\
\hline $\begin{array}{l}\text { Mean age at surgery } \pm \text { SD } \\
\text { (years) }\end{array}$ & $4.2 \pm 3.2$ & $4.3 \pm 3.4$ & $3.4 \pm 2.2$ & 0.098 \\
\hline \multicolumn{4}{|l|}{ Gender } & \multirow[t]{3}{*}{0.011} \\
\hline Female & $81(83.5)$ & $73(90.1)$ & $8(9.9)$ & \\
\hline Male & $16(16.5)$ & $10(62.5)$ & $6(37.5)$ & \\
\hline Circumcised males & $4(25)$ & $2(50)$ & $2(50)$ & 0.604 \\
\hline \multicolumn{4}{|l|}{ VUR grade } & \multirow[t]{6}{*}{0.049} \\
\hline $\mathrm{I}$ & $8(8.2)$ & $7(87.5)$ & 1 (12.5) & \\
\hline II & $14(14.4)$ & $12(85.7)$ & $2(14.3)$ & \\
\hline III & $49(50.5)$ & $46(93.9)$ & $3(6.1)$ & \\
\hline IV & $19(19.6)$ & $14(73.7)$ & $5(26.3)$ & \\
\hline $\mathrm{V}$ & $7(7.2)$ & $4(57.1)$ & $3(42.9)$ & \\
\hline \multicolumn{4}{|l|}{ VUR laterality } & \multirow[t]{3}{*}{0.515} \\
\hline Unilateral & $43(44.3)$ & $36(83.7)$ & $7(16.3)$ & \\
\hline Bilateral & $54(55.7)$ & $47(87.1)$ & $7(12.9)$ & \\
\hline \multicolumn{4}{|l|}{ Collecting system } & \multirow[t]{3}{*}{0.222} \\
\hline Single & $82(84.5)$ & $72(87.8)$ & $10(12.2)$ & \\
\hline Duplex & $15(15.5)$ & $11(73.3)$ & $4(26.7)$ & \\
\hline \multicolumn{4}{|l|}{ BBD } & \multirow[t]{3}{*}{0.282} \\
\hline Present & $27(32.5)$ & $22(81.5)$ & $5(18.5)$ & \\
\hline Absent & $56(67.5)$ & $51(91.1)$ & $5(8.9)$ & \\
\hline
\end{tabular}

BBD: bladder and bowel dysfunction; SD: standard deviation; VUR: vesicoureteral reflux.

Table 2. Univariate analysis of predictors of radiologic success 


\begin{tabular}{|l|c|c|c|c|}
\hline & $\mathbf{( n = 9 7 )}$ & Success (n=83) & Failure (n=14) & p \\
\hline Preoperative factors & & & & \\
\hline VUR grade & & & & 0.010 \\
\hline Low (I-III) & $71(73.2)$ & $65(91.5)$ & $6(8.5)$ & \\
\hline High (IV-V) & $26(26.8)$ & $18(69.2)$ & $8(30.8)$ & \\
\hline Intraoperative factors & & & & \\
\hline Volume of bulking agent injected & & & & 0.199 \\
\hline$<1 \mathrm{~mL}$ & $36(43.9)$ & $29(80.6)$ & $7(19.4)$ & \\
\hline$\geq 1$ mL & $46(56.1)$ & $42(91.3)$ & $4(8.7)$ & \\
\hline Intraoperative mound morphology & & & & 1.000 \\
\hline Excellent & $81(83.5)$ & $69(85.2)$ & $12(14.8)$ & \\
\hline Other & $16(16.5)$ & $14(87.5)$ & $2(12.5)$ & \\
\hline Postoperative factors & & & & \\
\hline Ultrasound mound visualization & & & & $<0.001$ \\
\hline Mound present & $85(87.6)$ & $81(95.3)$ & $4(4.7)$ & \\
\hline Mound absent & $12(12.4)$ & $2(16.7)$ & $10(83.3)$ & \\
\hline Combined classification model & & & & $<0.001$ \\
\hline Favourable & $70(72.2)$ & $68(97.1)$ & $2(2.9)$ & \\
\hline Unfavourable & $27(27.8)$ & $15(55.6)$ & $12(44.4)$ & \\
\hline
\end{tabular}

Table 3. Sensitivities and specificities of radiologic success based on postoperative ultrasound mound

\begin{tabular}{|l|c|c|c|c|}
\hline Study & Study period & $\begin{array}{c}\text { Sample size } \\
\text { (ureters) }\end{array}$ & Sensitivity (\%) & $\begin{array}{c}\text { Specificity } \\
\text { (\%) }\end{array}$ \\
\hline This study & $2008-2013$ & 97 & 98 & 71 \\
\hline Ellsworth et al & $2003-2008$ & 46 & 63 & 28 \\
\hline McMann et al. $^{25}$ & $2001-2005$ & 612 & 90 & 27 \\
\hline Park et al $^{28}$ & $2008-2009$ & 36 & 73 & 44 \\
\hline Kajbafzadeh et al $^{30}$ & $2003-2010$ & 341 & 94 & 22 \\
\hline Lee et al $^{12}$ & $2005-2010$ & 149 & 80 & 50 \\
\hline Ozcan et al & 26 & 36 & 44 & 50 \\
\hline Zamilpa et al & $1996-2003$ & 261 & 38 & 67 \\
\hline Zambaiti et al & $2005-2008$ & 53 & 100 & 66 \\
\hline
\end{tabular}

Table 4. Statistical performance of IMM, PUM and the combined classification model Variables Sensitivity (\%) $\quad$ Specificity (\%) PPV NPV 


\begin{tabular}{|l|c|c|c|c|}
\hline Excellent IMM & 83.1 & 14.3 & 85.2 & 12.5 \\
\hline Present PUM & 97.5 & 71.4 & 96.4 & 83.3 \\
\hline Favourable - combined model & 81.9 & 85.7 & 97.1 & 44.4 \\
\hline
\end{tabular}

IMM: intraoperative mound morphology; NPV: negative predictive value; PPV: positive predictive value; PUM: postoperative ultrasound mound. 